\title{
Parosteal Osteosarcoma
}

National Cancer Institute

\section{Source}

National Cancer Institute. Parosteal Osteosarcoma. NCI Thesaurus. Code C8969.

A low grade malignant bone-forming mesenchymal neoplasm arising from the surface of the bone. It usually affects the distal posterior femur, the proximal tibia, and proximal humerus. Painless swelling is the usual clinical sign. Most patients are young adults and the prognosis is usually excellent. 